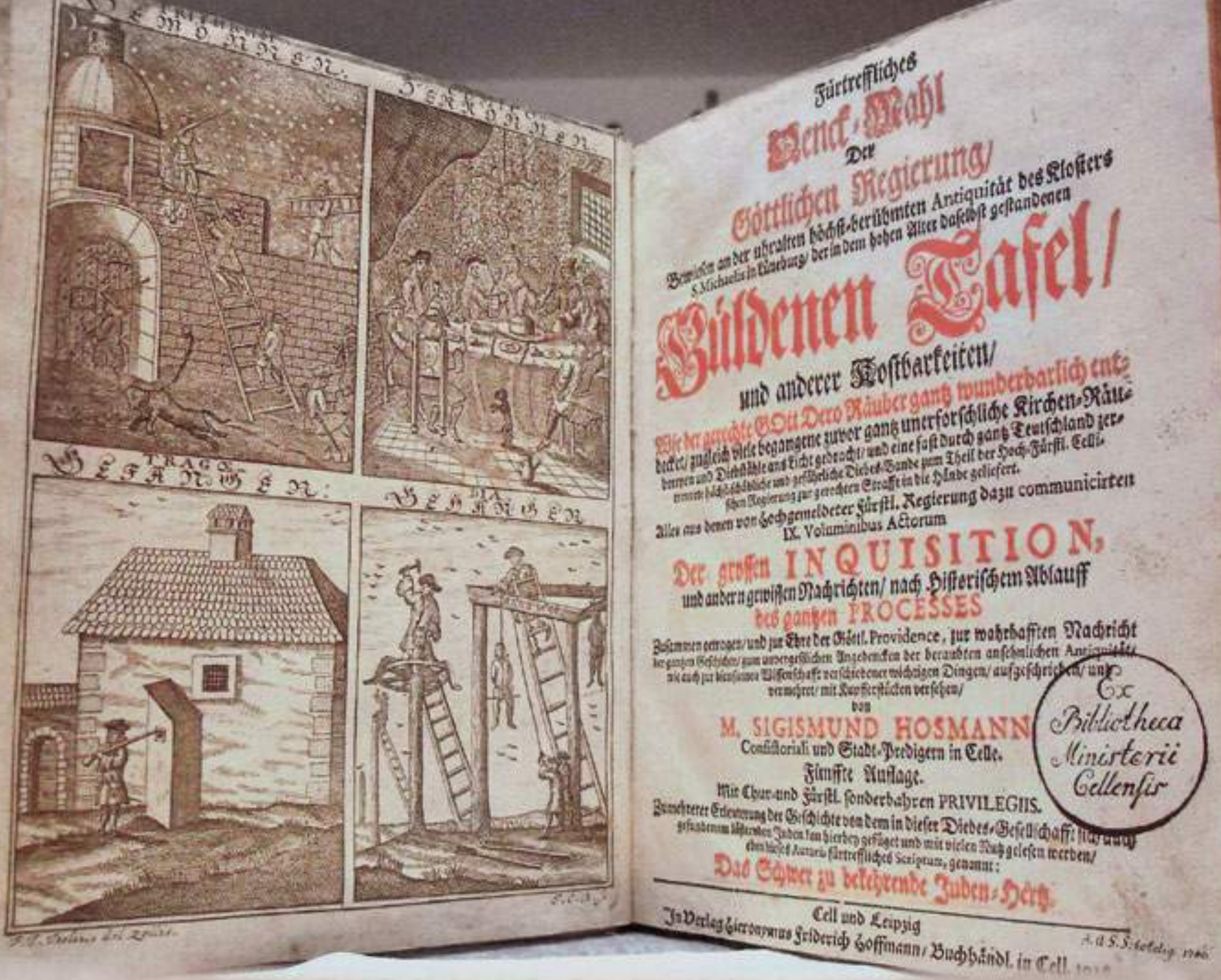

Sigismund Hosmann: Fürtreffliches Denck=Mahl, Celle/Leipzig 1700, Vorsatz und Titelblatt

\title{
Auf den Spuren des Räubers Nicol List (1654-1699)
}

\author{
Gerd Freitag
}

1 http://www.zehn.de/nikollist-6997623-5/

Letzter Zugriff 22.7.2015

In einer Auflistung der legendärsten deutschen Räuber im Internet wird der sächsische Meisterdieb und Hochstapler Johann Nicolaus List, besser bekannt als Nicol oder Nickel List, an fünfter Stelle genannt. ${ }^{1}$ In der Literatur finden sich zahlreiche weitere Rufnamen und Pseudonyme wie z.B. Niklas Liste, Freiherr Johann Rudolph von der Mosel, Baron aus Sachsen, Doktor aus Halle, Schwarzer Nickel, Grätzischer Nickel oder Nicol Weichel. Beim Weitersuchen findet man eine erstaunlich große Anzahl von Publikationen von Schriftstellern, Journalisten, Pfarrern, Wissenschaftlern, Regionalforschern, Künstlern u. a. aus verschiedenen deutschen Ländern, die sich seit mehr als 300 Jahren bis in die jüngste Gegenwart mit dem Leben und den (Un-)Taten dieses Mannes beschäftigt haben oder beschäftigen. Der Versuch einer Bibliografie weist mehr als 350 Einträge auf: Archivakten, Historische Romane, Publikationen in Kalendern, Zeitungen und Zeitschriften, CDs, Theaterstücke, Balladen, Sagen, Gedichte, ein Kinderbuch, eine Kinderoper, Gemälde und Lithografien, Spiele usw.

Neue Erkenntnisse zu einem Münzfund 1939 im Dorf Brünlos, heute Ortsteil von Zwönitz, veranlassten den Autor, nach möglichen früheren Besitzern zu recherchieren. Dabei kam 
der Räuber Nicol List ins Blickfeld, dessen frühere Wirkungsstätte um Hartenstein nur wenige Kilometer von der Vergrabungsstätte entfernt lag. Auch das Zeitfenster passte in etwa. Der Beweis auf einen Zusammenhang mit List wurde bisher nicht erbracht. Das Interesse am Räuber List war geweckt.

Die zahlreichen Archivalien und Publikationen über List in Archiven, Museen, Bibliotheken und Redaktionen in den Bundesländern Sachsen, Thüringen und Niedersachsen sowie im Internet wurden in sechsjähriger Forschungsarbeit in Zusammenarbeit mit der Sächsischen Landesstelle für Museumswesen Chemnitz gesichtet, ausgewertet und ein Lebenslauf sowie eine Bibliografie erstellt. Dazu wurden mehrere Anlagen erarbeitet, darunter eine Zeittafel, eine Zusammenstellung von Zeitdokumenten sowie der etwa 120 namentlich bekannten Komplizen, eine Sammlung der Balladen, Sagen und Gedichte sowie von Abbildungen.

Es fällt ins Auge, dass zwischen dem archivalisch überlieferten Lebenslauf Lists und den in den Printmedien und im Internet veröffentlichten Biografien z. T. erhebliche Unterschiede bestehen. Die meisten Autoren, darunter auch die Herausgeber großer historischer Lexika wie Zedler und Pierer, übernahmen ungeprüft einen Lebenslauf, der erstmals 1700 von dem Celler Stadtprediger Magister Sigismund Hosmann in seinem Buch „Fürtreffliches Denck=Mahl der Göttlichen Regierung Bewiesen an der uhralten höchst=berühmten Antiquität des Klosters S. Michaelis in Lüneburg / der in dem hohen Altar daselbst gestandene Güldene Taffel / und anderer Kostbarkeiten / wie der gerechte Gott Dero Räuber gantz wunderbahrlich entdecket / zugleich viele begangene zuvor gantz unerforschliche Kirchen=Räubereyen und Diebstähle ans Licht gebracht / und eine fast durch gantz Teuschland zertrennete höchst=schädliche und gefährliche Diebes= Bande zum Theil der Hoch=Fürstl. Zellischen Regierung zur gerechten Straffe in die Hände geliefert" veröffentlicht wurde.

Das Buch war ein Bestseller des 18. Jahrhunderts. Es erschien nach dem Tod Hosmanns (1701) in weiteren fünf deutschen und einer niederländischen Ausgabe. Hosmann hatte seit Januar 1699 als Seelsorger Nicol List und mehrere Komplizen im Gefängnis Celle betreut. Er hatte ihn am 23. Mai 1699 zur Hinrichtung begleitet und außerdem Zugang zu den Prozessakten. Deshalb waren die nachfolgenden Autoren mit Ausnahme mehrerer sächsischer Lokalforscher, die die Harten- steiner Gerichtsbücher ausgewertet hatten², davon überzeugt, dass Hosmanns Buch authentisch und überprüfbar das Leben und Wirken Lists und seiner Komplizen widerspiegeln würde.

Das ist jedoch m. E. nicht der Fall. Nach gründlicher Kenntnis der Aktenlage zu List und seinen Komplizen im gesamten Wirkungsraum des Räubers ist einzuschätzen, dass Hosmanns „Fürtreffliches Denck-Mahl“ zwar eine wichtige, aber keine vollständige und in jeder Hinsicht authentische, wissenschaftlich fundierte Quelle für die Aufklärung und Bewertung des Lebens und Wirkens Lists u. a. ist. Deshalb kann sie auch nicht kritiklos unter dem Begriff einer „Aktenmäßigen Überlieferung“ eingeordnet werden $^{3}$.

Hosmann hat etwa ein Jahr nach Lists Hinrichtung seine Erinnerungen aus dem Gedächtnis sowie aus Erkenntnissen der Inquisition niedergeschrieben. Ihm lagen vermutlich keine Dokumente und präzise Zeugenaussagen über Geburt, Familie, Kindheit und Jugend Lists, seine berufliche Entwicklung sowie Details über seinen Militärdienst vor. Beginnend beim falschen Geburtsjahr 1656 (List wurde 1654 geboren) sind die Angaben Hosmanns über Familie, Schulzeit und Ausbildung Lists, seine angebliche Dienstzeit in der kurbrandenburgischen Armee, die Teilnahme an Schlachten 1675 gegen die Schweden und 1679 gegen die Franzosen nicht belegbar. Hosmanns mehrmals anzutreffende Behauptung, List habe nach seiner Militärzeit einen Gasthof in Ramsdorf bei Borna gekauft und betrieben, der sich rasch zu einem Räubernest entwickelt habe, gehört ebenfalls in das Reich der Legende. Vermutlich war hier ein Gasthof im Dorf Raum bei Beutha im Erzgebirge gemeint. ${ }^{4}$

Überhaupt spiegelt das Hosmannsche Buch wider, dass der Autor keine gesicherten Kenntnisse über das Vorleben Lists in Sachsen zwischen 1654 und 1696 hatte und diesen Zeitraum nach eigenem Ermessen literarisch füllte. Hinzu kommen eine Reihe Ungenauigkeiten in den Texten, die vermutlich aus Hör- oder Druckfehlern resultieren. Das betrifft vor allem Personen- und Ortsangaben sowie Kalenderdaten. Der häufige Gebrauch von Spitznamen bzw. Tarnnahmen Lists und der Komplizen erschwert teilweise ihre Identifizierung. Der verübte Einbruch beim Erbrichter Hilbert fand nicht in Richerswalde, sondern im erzgebirgischen Kleinrückerswalde bei Annaberg statt. Die
2 Insbesondere die sächsischen Lokalforscher Richard Hommel, Oberlungwitz 1925; Prof. D. Dr. Otto Clemen, Zwickau 1927; Richard Oertel, Aue 1934, 1935; Isodor Hottenroth, Gersdorf 1937 und Albin Schwind, Beutha 1940, machten sich durch die Auswertung der Hartensteiner Gerichtsbücher der Jahre 1688 bis 1699 und eigene Forschungen um die List-Biografie verdient.

3 Neben den Strafakten in mehreren Archiven liegen zum Kriminalfall List zwei „Aktenmäßige Überlieferungen“ vor: „Historische Ausführliche und glaubwürdige Erzehlung [...]", erschienen 1699 in Celle und Leipzig, und „Ausführliche Relation, wie es bei der scharffen Execution, derer in Zelle [...] wuercklich vollenzogen [...]“. Celle 1699. Beide verfasst von anonymen Autoren, die wahrscheinlich selbst dem Inquisitionsgericht in Celle angehört hatten. Sie können als authentisch und überprüfbar angesehen werden, umfassen aber nur einen kleinen Ausschnitt aus den Strafverfahren gegen List.

4 Vielleicht umgangssprachlich Raumsdorf statt Ramsdorf? Im Historischen Ortsverzeichnis von Sachsen gibt es diese Ortsbezeichnung jedoch nicht. 
Moderne Illustration zu Nicol List, Zeichnung von Heinz Bormann aus dem „Magazin", 1963

Sigismund Hosmann: Für treffliches Denck=Mahl, Celle/Leipzig 1700, Teil 1, S. 165-166.

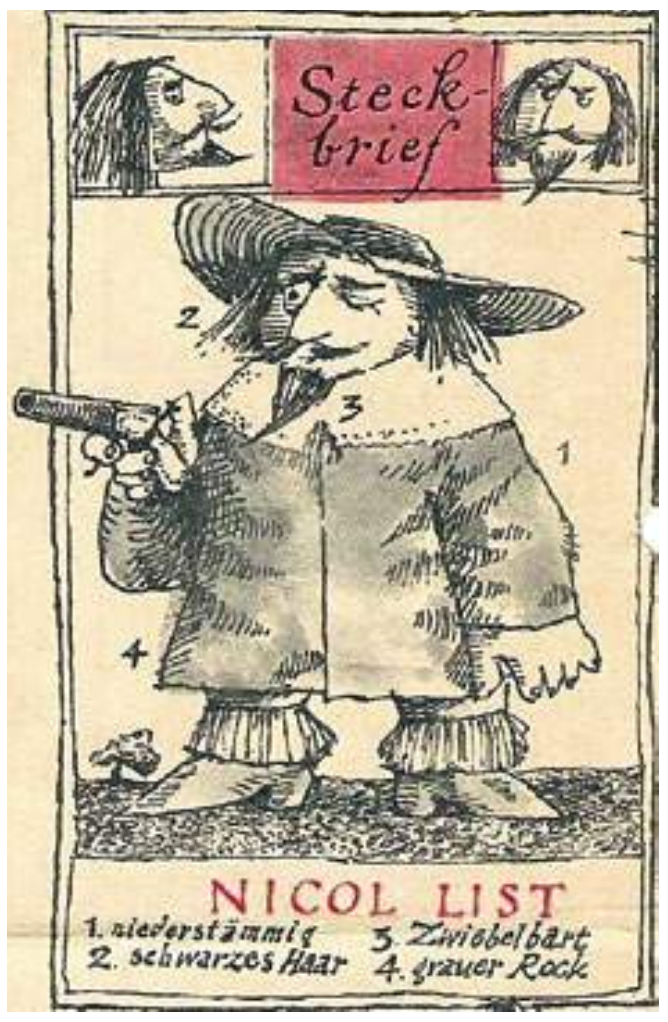

Wüstung des List'schen Hauses in Beutha erfolgte nicht 1700 aufgrund des explodierenden Volkszornes der Dorfbewohner, sondern 1696 aufgrund einer schriftlich von der Schönburger Herrschaft angewiesenen Strafmaßnahme usw.

Bei den Zeitangaben muss berücksichtigt werden, dass in Sachsen 1700 die Zeitumstellung vom Julianischen auf den Gregorianischen Kalender mit einer Zeitverkürzung von 11 Tagen (auf den 18. Februar folgte der 1. März) vorgenommen wurde und andererseits ein Teil der überlieferten Zeitangaben nach den Bezeichnungen des Kirchenjahres dokumentiert ist.

Hosmann hatte seine Grenzen selbst erkannt, wie er seinem Buch mitteilte: „Allein hier bedaure ich bey seinen Leb=Zeiten / nicht habe vorher wissen mögen / daß ich diese Geschichte / und in selbiger seinen Lebens=Lauf schreiben mögen. Es waren das zu der Zeit meine allerwenigsten Gedanken. Ich hätte sonst vieles von ihm und aus seinem Munde anmercken können / indem ich manchmahl ganze Stunden bey ihm im Gefängnis gesessen / da er wann ich meine geistige Unterredung mit ihm geschlossen / gar gerne aus seinem Leben erzehlte / welches ich aber so groß nicht achtete / so fern ess nicht zu meinem damahligen Zweck gereichte / der allein auf Erkäntnis seiner Sünden und andere zur Auffheissung seiner tieffster Reue zerknirrschten Seele nöthige Dinge an- ging. Muß also nur das erzehlen, wessen ich mich noch erinnern kan / und was ich nechst dem in dem ganzen Corpore Actorum hin und wieder gefunden / und als kleine Stücklein und Brosamen zusammen getragen habe / auch was ich sonst aus andern gantz glaubhafften Berichten erfahren können." ${ }^{\text {" }}$

Eine Reihe von historischen Universal-Lexika bezieht sich ausdrücklich als Quelle auf Hosmanns „Fürtreffliches Denck=Mahl“ und gibt somit die lückenhaften, ungenauen bzw. falschen Inhaltsangaben zum Lebenslauf Lists wieder. Beispielhaft sei hier das „Zedlersche Universal-Lexikon“, Band 17, S. 1635 1646, erwähnt: Der umfangreiche Eintrag beginnt mit den Worten: „List, (Nicol) oder wie er sich sonst nannte, Johann Rudolph von der Mosel war um das Jahr 1656 gebohren“. Noch drastischer sind die Abweichungen in „Pierers Universal-Lexikon“, Altenburg 1860, Band 11, S. 880. In diesem modernen allgemeinen Lexikon, das zum Vorbild für die großen Lexika wie Brockhaus, Meyer und Herder wurde, summieren sich falsche Tatsachenbehauptungen über Lists Lebenslauf, beginnend mit dem Geburtsjahr 1656 und dem angeblichen Geburtsort Eichlaide in Sachsen - dieses Dorf wurde erst um 1700 gegründet. Seine frühen Dienstjahre werden gar an einen gräflich reußischen Hof und sein erster Gasthof nach Reinsdorf bei Zwickau verortet. Ähnlich kritisch sind auch die Einträge zu List in etwa einem Dutzend weiterer Lexika und biografischen Sammlungen $\mathrm{zu}$ bewerten, die in das Internet übernommen worden sind. Analog sind die Eintragungen in den zahlreichen historischen Kalendern, Zeitschriften usw. einzuschätzen.

Spätere Autoren, besonders im 18. Jahrhundert, haben in einer großen Zahl biografischer Romane und in einer unüberschaubaren Flut von Beiträgen in den Printmedien Nicol List zum Räuberhauptmann und gar zum literarischen Vorbild für den Karl von Moor in Schillers „Die Räuber“ hochstilisiert. Tatsächlich war List ein mit allen Wassern gewaschener charismatischer Meisterdieb und Hochstapler, ein Gleicher unter Seinesgleichen, der nur ein Ziel kannte: Er wollte ein möglichst großes Stück vom Kuchen abhaben, mit dem die Tische der barocken Obrigkeiten seiner Zeit reichlich gedeckt waren. Da ihm das seine Herkunft und sein Werdegang verweigerten, griff er zur Selbsthilfe und bewegte sich dabei mit wechselndem Erfolg in einem ganzen Netz von Gaunern, aus dem er lediglich als der „Vornehmste“ und handwerklich Geschickteste herausragte. Es 
gab nie eine einheitliche, straff organisierte Bande, sondern eine Reihe von mehr oder weniger selbständig handelnden Diebesrotten mit Kristallationskernen im sächsischen Vogtland, in Stedten (Thüringen), Halle, Altona bei Hamburg, Meuslingen bei Lübeck sowie Blumenau und Wunstorf bei Lüneburg.

Für den Autor ergibt sich aus dem vorliegenden Sachverhalt die Frage, wie Internetnutzer mit den anhand der Archivdokumente nachweisbar ungenauen bzw. falschen historischen Überlieferungen umgehen sollten, da sie bisher kaum die reale Möglichkeit haben, eine kritische Bewertung anhand eigener gesicherter Erkenntnisse vornehmen zu können.

Eine umfassende, auf gründliche Archivstudien gestützte Biografie des Nicol List gibt es bisher nicht. Diese wird gegenwärtig vom Autor erarbeitet. In Kurzfassung lassen sich folgende Fakten aus dem Lebenslauf des Nicol List nachweisen:

Der im sächsischen Waldenburg geborene und am 4. Dezember 1654 getaufte Sohn einer Tagelöhnerfamilie wuchs in den Ställen der Schönburger Herrschaft rund um Hartenstein auf und entwickelte sich zum Reitknecht und gefragten Pferdekenner. 1679 heiratete er die aus Thierfeld stammende Maria Scherf und bezog mit ihr ein Häuschen in Beutha, wo er ab 1680 als Bürger geführt wurde. 1681 trat er in den Militärdienst ein, wurde Kürassier der kurfürstlich sächsischen Armee und diente als Korporal u. a. in Wittenberg, in der Lausitz, im Meißnischen Kreis und zuletzt bei Freiberg. 1683 verstarb seine erste Ehefrau an der Geburt der gemeinsamen Tochter Anna Marie. 1686 nahm er als Kürasier mit einem Kontingent der kurfürstlich sächsischen Armee an der kaiserlichen Schlacht gegen die Türken um Ofen (heute Teil von Budapest) in Ungarn teil. Aus dem Krieg unversehrt zurückgekehrt, betrieb er seine Entlassung aus dem Militärdienst. Sein Vorgesetzer verweigerte ihm zunächst den „Ehrlichen Abschied“ und forderte als Gegenleistung zwei Pferde und 30 Taler. List verließ 1687 eigenmächtig seine Garnison in Langenau unter Zurücklassung seines Pferdes und seiner gesamten Ausrüstung, kaufte in Beutha einen Bauplatz und baute ein neues, größeres Haus. Er betrieb Gastwirtschaft und Branntweinbrennerei sowie mit Schönburger Pass landesweit und nach Böhmen Pferdehandel. Die Auseinandersetzung um den „Ehrlichen Abschied“ zog sich mehrere Jahre hin. List wurde des-

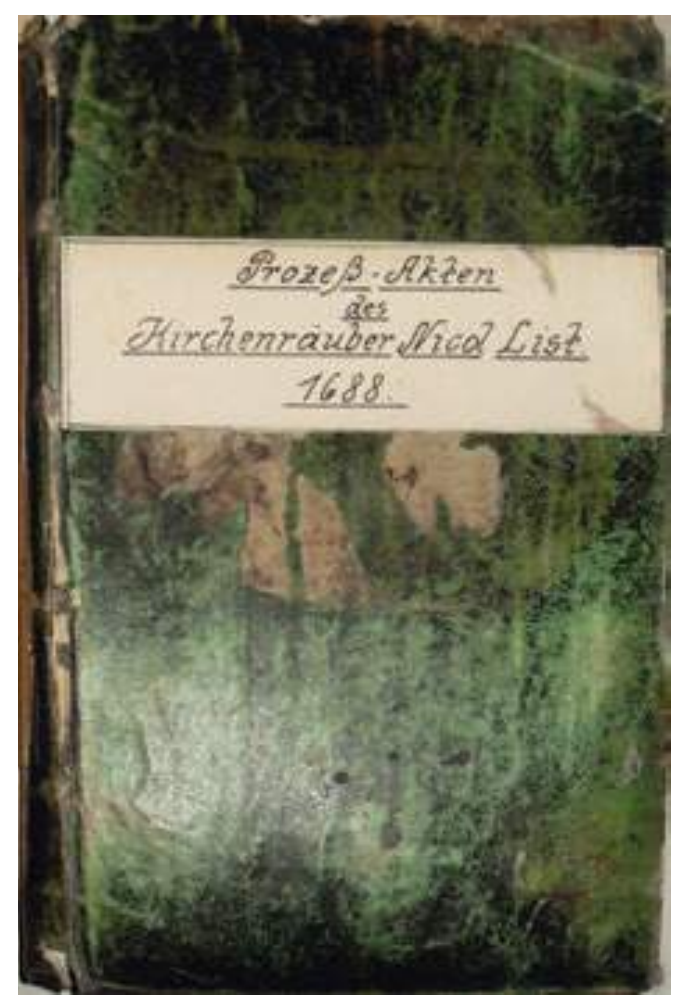

Gerichtsbuch der Herrschaft Hartenstein mit Prozessakten, betreffend den Räuber Nicol List, 1688 und folgende Jahre halb wiederholt von kursächsischen Soldaten bedrängt und ausgeraubt. Der Versuch, ihn zwangsweise wieder in den Militärdienst zu pressen, scheiterte, bis schließlich eine Einigung mit dem Rittmeister gegen die Zahlung von Geld und Pferden erzielt werden konnte.

List hatte aus dem Krieg eine neue Frau mit nach Beutha gebracht, Margarethe Göden (Göthin) aus Burg bei Magdeburg. Nach zwei Jahren „wilder Ehe“ durch Weisung der Obrigkeit gegen seinen Willen zur Eheschließung gezwungen, hatte er mit ihr fünf gemeinsame Kinder, von denen eines im Kindesalter verstarb.

List verstand es lange Zeit, sich in der Öffentlichkeit den Anschein einer gebildeten, weltmännisch erfahrenen und finanziell gut situierten Persönlichkeit zu geben. Dazu trugen auch sein häufiges Selbststudium von Fachliteratur über Astronomie und Medizin sowie sein Ruf als Doktor von Mensch und Tier bei. Er pflegte freundschaftlichen Umgang mit Angehörigen der Schönburger Herrschaft und konnte mehrere von ihnen als Paten für seine Kinder gewinnen. Darunter war eine Tochter des damals höchsten Beamten in Hartenstein, des Amtsverwesers Schönheim, sowie ein Lößnitzer Ratsherr. List war auch selbst ein gefragter Pate. Gegenüber den Ämtern trat er selbstbewusst auf und stützte sich im Konfliktfall auf mehrere Advokaten. Das brachte ihm die Anerkennung seitens seiner Geschäftspartner, Bediensteten und der Dorfbewohner ein. 


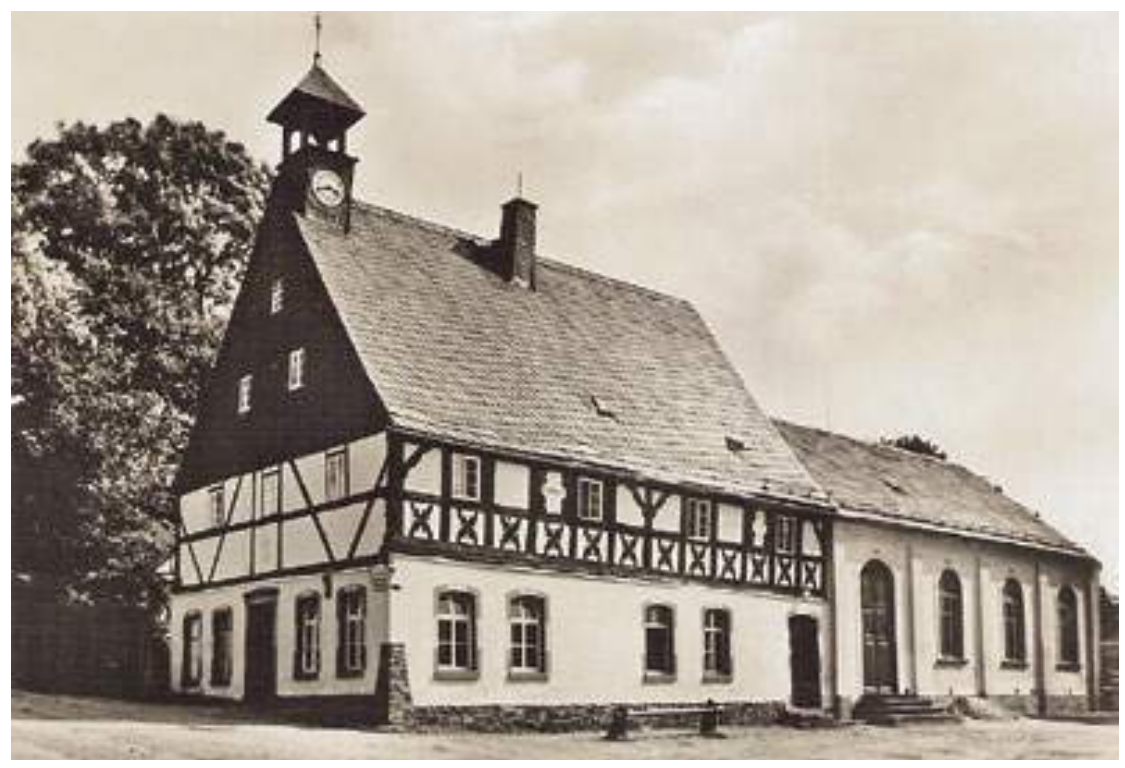

„Grüne Tanne“ in Raum, 1966

Beutha, Erinnerungssteine an der Friedhofsmauer
1691 übernahm List als Pächter der Schönburger Herrschaft den neugebauten Straßengasthof „Zur grünen Tanne“ im Nachbarort Raum, der sich bald zum Räubernest entwickelte. Dabei spielten ständige Auseinandersetzungen mit aktiven sowie unehrlich entlassenen kursächsischen Soldaten, die Lists Frau auch in seiner Abwesenheit drangsalierten und beraubten, eine besondere Rolle. Aufgrund vieler Beschwerden beim Amt musste List nach 17 Monaten die Pacht beenden. Er ging 1693 in seine Wirtschaft nach Beutha zurück und war als Pferdehändler viel unterwegs.

Verdachtshinweise darauf, dass List in der Vergangenheit Straftaten begangen hatte und u. a. an Pferdediebstählen beteiligt war, wurden im Hartensteiner Amt mehrmals bekannt, jedoch nie nachgewiesen. 1692 war er an einem spektakulären Einbruch bei einer Frau von Tettau auf dem Gut in Mechelsgrün bei Plauen im Vogtland beteiligt. Sein Beuteanteil betrug 1.200 Taler. Daraus entwickelte sich in der Folgezeit ein heftig geführter Beutestreit, der zu harten Drangsalierungen Lists und seiner Frau durch seine Kumpane führte. Der Beuteanteil wurde ihm letztlich wieder abgepresst.

1694 war List als Anführer einer Rotte von Soldaten aus der Garnison Zwickau an einem Einbruch bei einem Freiherrn von Meusbach im Schloss Braunsdorf im Fürstentum Sachsen-Zeitz beteiligt. Gegen die Soldaten wurden Strafverfahren durchgeführt, während List unbehelligt blieb. Gerüchteweise wurde mehrmals die Vermutung geäußert, dass List vom Hartensteiner Amt gedeckt werde.

Im Ergebnis eines heftigen Beutestreites zwischen Mittätern wurde 1695 in Lists Scheune in Beutha der „Wachtmeister“ genannte Komplize Eckardt erschlagen, sein Kopf abgetrennt und der Körper in einen Teich in Jüdenhain bei Mosel geworfen, wo er später gefunden wurde. Obwohl die Tat in Beutha mehreren Personen unverzüglich bekannt wurde, erfolgt keine Strafverfolgung der Täter. List war Mitwisser, aber angeblich an dem grausamen Mord nicht selbst beteiligt. 1696 führte List mit vier Komplizen einen Einbruch bei dem reichen Erbrichter Hil-
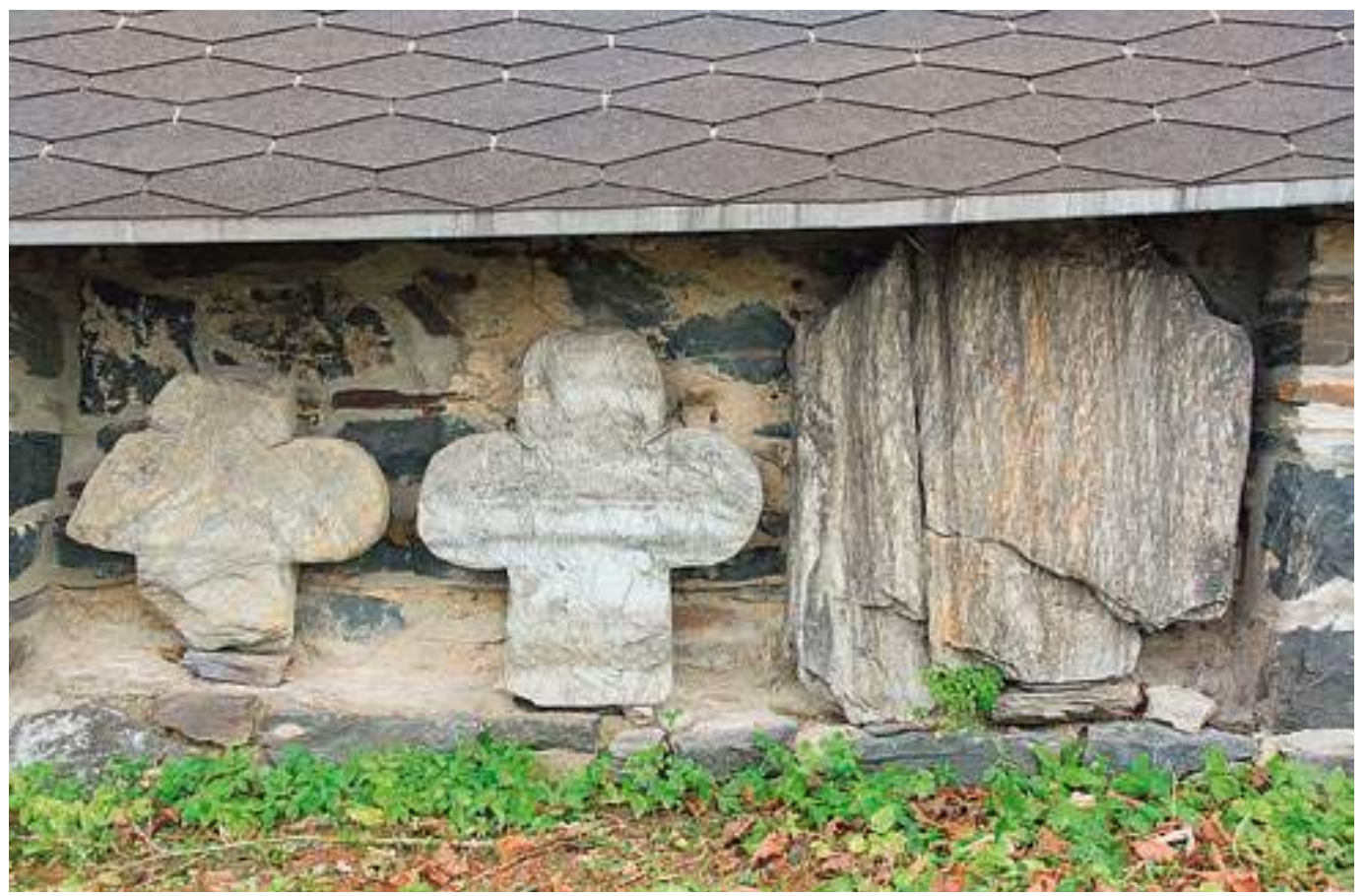
bert in Kleinrückerswalde bei Annaberg durch, bei dem erhebliche Beute gemacht wurde. Sofortige Nachforschungen des Richters führten sehr schnell auf die Spuren der Täter und zur Person von Nicol List als Anführer. Hilbert drängte auf sofortige Verhaftung Lists. Zur Ausführung wurden zwanzig Personen unter dem Kommando des Hartensteiner Landrichters Zechendörfer eingesetzt, die List um Mitternacht in Beutha festsetzen sollte. Durch das unentschlossene Handeln des Landrichters schöpfte List Verdacht, gab zwei Pistolenschüsse ab und ergriff die Flucht auf seinem Pferd. Die Schüsse töteten einen Landschöppen und verletzten einen zweiten Verfolger so stark, dass dieser nach wenigen Stunden verstarb.

Das vom Amt Hartenstein eingeleitete Inquisitionsverfahren wegen zweifachen Mordes führte nicht zum Ergebnis. Der Aufenthalt von List konnte trotz zahlreicher Steckbriefe und vieler Zeugenhinweise nicht festgestellt werden. List wurde schließlich unter Ober-Acht gestellt, also für vogelfrei erklärt, was nach deutschem Recht ein Jahr, sechs Wochen und drei Tage andauerte. 1696 wurde auf Weisung der Schönburger Herrschaft Lists Haus in Beutha abgerissen, 1700 wurden an diese Stelle zur Mahnung an die Bluttat drei Erinnerungssteine gesetzt, deren Texte das Tatgeschehen wiedergaben. Die Steine sind heute

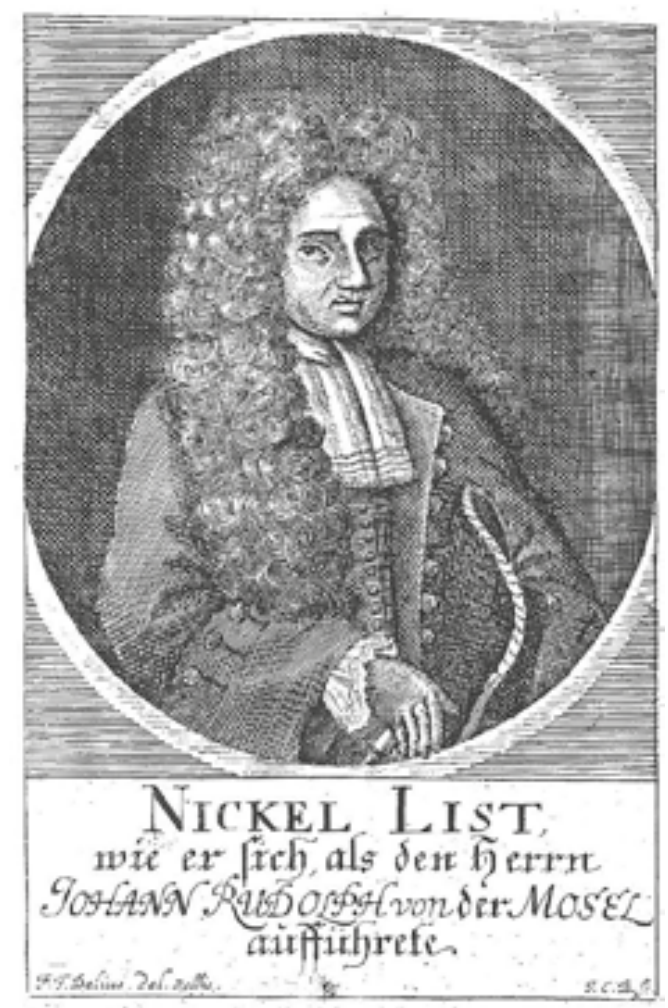

an der Innenseite der Friedhofsmauer in Beutha aufgestellt, die überlieferten Texte darauf jedoch nicht mehr lesbar.

List hatte sich nach seiner Flucht 1696 aus Beutha u. a. in Wittenberg, Fulda, Leipzig und Halle aufgehalten und von Pferdehandel und Wirtstätigkeit ernährt. In Leipzig und Halle traf List auf Angehörige mehrerer Diebesrotten, die sich u. a. auf Diebstähle zur Leipziger Messe spezialisiert hatten. Darunter waren auch Juden, die List bereits vor Jahren in Beutha als reisende Händler kennengelernt hatte und die sich insbesondere als Hehler betätigten. Diese unterhielten Verbindungen zu Diebesrotten in Altona bei Hamburg, Meuslingen bei Lübeck und Blumenau und Wunstorf bei Lüneburg. 1696 und 1697 nahm List an einer Serie von Einbrüchen bei reichen Kaufleuten, Grundherren, Pfarrern und in Kirchen, darunter dem Naumburger Dom, teil.

Durch seine „Erfolge“ hatte sich List einen sagenhaften Namen als außergewöhnlich begabter Nachschlüssel-Spezialist gemacht, der in der Lage sei, „auf leisen Sohlen“ jedes Schloss zu öffnen, gleich welchen Schwierigkeitsgrades. Man sagte ihm nach, dass er Zauberei betreiben und durch Wände gehen könne. Ein Altonaer Komplize holte den legendären „sächsischen Baron“ nach Hamburg, wo im Dom angeblich eine Tonne Gold läge. List führte den Einbruch mit mehreren Komplizen aus, war aber über das Ergebnis enttäuscht.

In Hamburg lernte List weitere Komplizen kennen, die an künftigen Diebstählen beteiligt waren. Dazu zählte die getrennt lebende Ehefrau eines bankrotten Weinhändlers, Anna von Sien, die List als seine künftige Konkubine auswählte. Über die Altonaer und Meuslinger Juden sowie über Anna von Sien entstanden die Beziehungen Lists zu einer Diebesrotte um den pensionierten Offizier Gideon Perrmann in Blumenau und Wunstorf.

In Niedersachsen schlüpfte List für die Öffentlichkeit in eine neue Rolle: Er nannte sich nun Freiherr Johann Rudolph von der Mosel und hatte mit der „Adligen aus Hollstein“ Anna von Sien, seinem persönlichen „Jäger“, dem wegen Totschlages gesuchten Weimaraner Andreas Schwarz, den „Bediensteten“ Christian und Gertrud Schwanke sowie dem Ehepaar Lorenz und Katharina Schöne einen eigenen „Hofstaat“. Sie quartierten sich in wechselnden Gasthöfen und Herbergen ein und genossen das Leben nach hochherrschaftlichem Vorbild in vollen Zügen. List
Nicol List als Johann Rudolph von der Mosel, Stich, 1700 


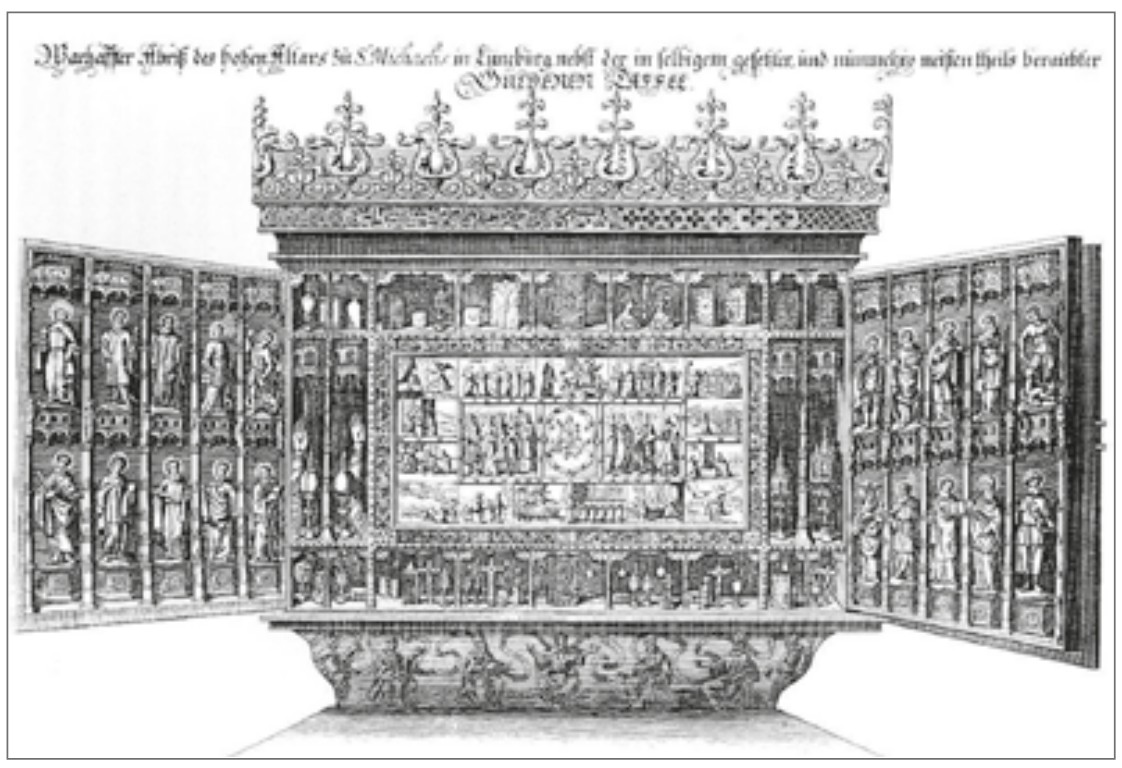

Goldene Tafel in Lüneburg Stich, um 1700

Nicol List im Gefängnis Stich, 1700 selbst verkleidete sich $u$. a. als Kaufmann mit einer langen braunen Allongeperücke und wertvoller Kleidung, verließ jedoch sein Herbergszimmer nur selten und immer in Begleitung seines „Jägers“.

List erhielt die Nachricht, dass in der Kirche St. Katharina in Braunschweig ein lukrativer Schatz zu heben sei: der Nachlass einer Generalswitwe, verpackt in zehn Koffern. Er führte den Raub im Januar 1698 mit mehreren Komplizen aus. Teile der Beute wurden ihm später als sichergestellte Beweismittel zum Verhängnis, dar-

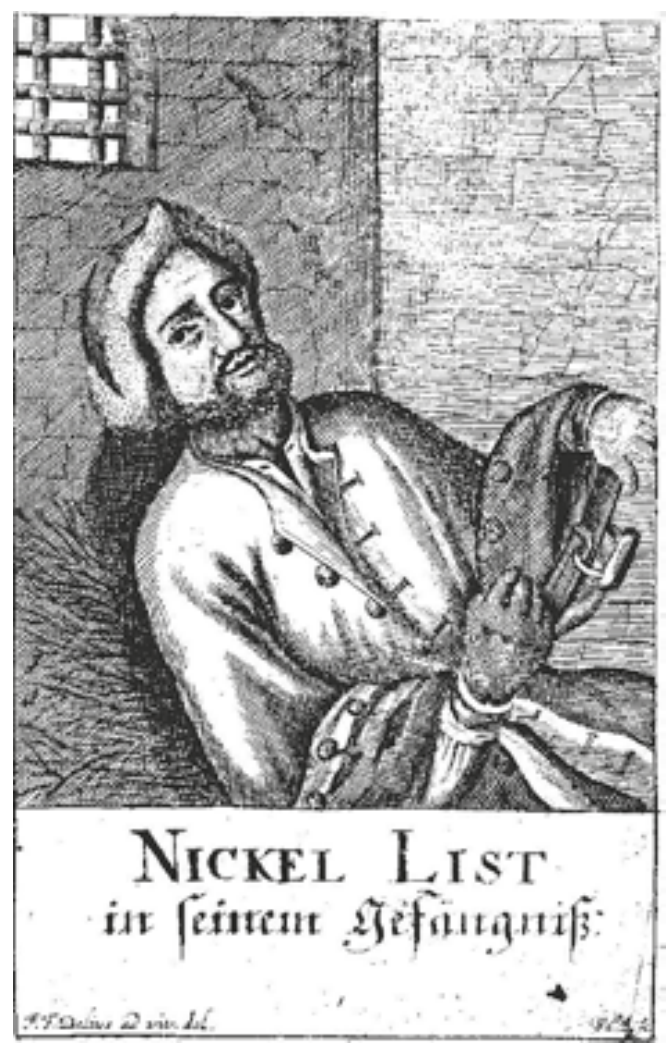

unter ein wertvoller Säbel und eine Kaminuhr.

In Hamburg hatten List und Schöne zufällig Kenntnis davon erhalten, dass in Lüneburg eine besonders wertvolle Goldene Tafel im Altar der Kirche St. Michaelis vorhanden sei und Mitglieder der Altonaer Rotte einen Raub derselben beabsichtigten. Sie verabredeten, den Altonaern zuvorzukommen, und führten die Tat nach gründlicher Vorbereitung im März 1698 aus. Der Einbruch wurde erst nach drei Tagen zufällig entdeckt. List und einige Komplizen hatten sich bereits in Richtung Sachsen und Thüringen abgesetzt und begingen auf dem Fluchtweg mehrere Kircheneinbrüche. Der Diebstahl der Goldenen Tafel ging aufgrund des historischen und materiellen Wertes als der größte Kirchenraub aller Zeiten in die Geschichte ein.

Mit vier ausgewählten Komplizen beging List im Juni 1698 einen Einbruch bei dem reichen Adjunkten Schmidt in Hof im bayrischen Vogtland. Mit dem Beutegut begaben sich die Reiter zum Landgasthof „Neu Schenke“ bei Daßlitz nahe Reichenbach im Vogtland. Zu nachtschlafener Zeit wurden sie dort von den Verfolgern aus Hof gestellt. List und zwei Mittäter wurden festgesetzt, zwei weitere entkamen. List verletzte durch Pistolenschüsse einen Verfolger, wurde aber beim Fluchtversuch niedergeschlagen und war nicht mehr zur Flucht im Stande. Daraufhin versuchte er, sich mit einem Barbiermesser die Kehle durchzuschneiden, was nur teilweise gelang. Ein anwesender Medicus konnte noch rechtzeitig einen tödlichen Ausgang verhindern.

Im Gefängnis Greiz der Herrschaft Reuß begannen die Ermittlungen durch die Inquisition, wobei zunächst nur der Einbruch bei Schmidt und mehrere Kircheneinbrüche im Vogtland Untersuchungsgegenstand waren. Kenntnisse über die Tötung der Bürger in Beutha und die Kircheneinbrüche in Niedersachsen lagen noch nicht vor.

Gerüchteweise gelangte die Information nach Hartenstein, dass Nicol List in Greiz einsitzen würde. Als sich dieser Hinweis durch Zeugen bestätigt hatte, reiste der Hartensteiner Amtsverweser Schönheim sofort selbst nach Greiz und verhörte List insbesondere zu den Tötungshandlungen in Beutha. List spielte den Reumütigen. Er versuchte die Sache als Unfall darzustellen, woran der Hartensteiner Landrichter schuld sei. Schönheim schien ihm zunächst Glauben zu schenken. Er vernahm in Hartenstein nochmals alle Tatzeugen, konnte jedoch die Unfallvari- 


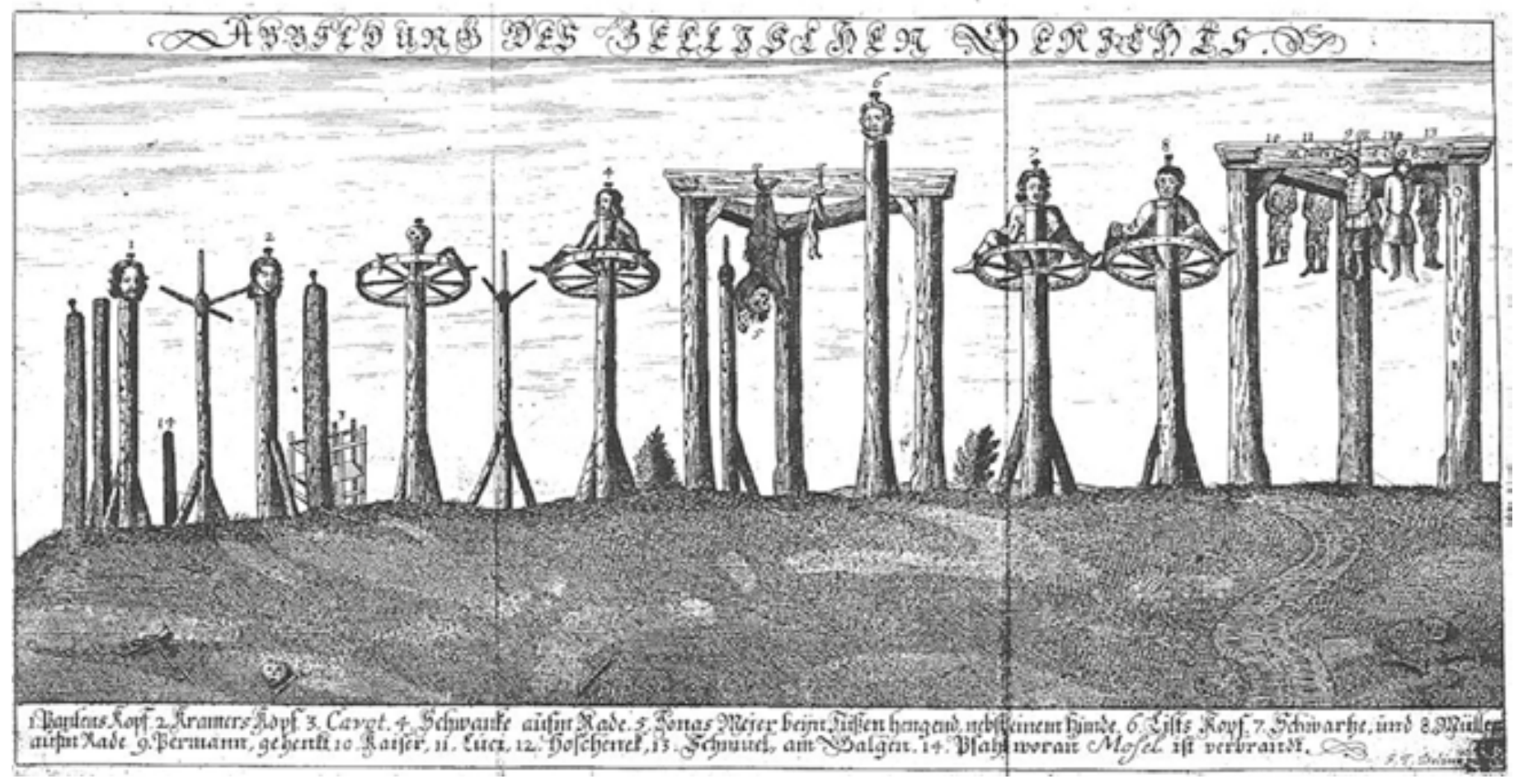

ante nicht bestätigen. Jetzt bemühte sich Schönheim im Auftrag der Schönburgischen Herrschaft intensiv darum, List nach Hartenstein überführen zu lassen. List und die beiden Komplizen wurden in das Gefängnis Hof überführt. Nach anfänglicher Zusage verzögerte Hof die Auslieferung. Nun führte die Inquisition der Markgrafschaft BrandenburgBayreuth die Ermittlungen zu Ende und verurteilte List am 29. Oktober 1698 zum Tode durch Schleifen durch unvernünftige Tiere zum Richtplatz und Lebendigverbrennen. Der Komplize Nicol Horn alias Hans Krause wurde gehängt, der Komplize Buttelstädt der Prügelstrafe unterzogen und des Landes verwiesen. Nachdem Schönheim den List zweimal im Gefängnis Hof aufgesucht und dessen verschärfte Bewachung veranlasst hatte, übergab er schließlich die Hartensteiner Akten an die Markgräflich-Bayreuthische Inquisition.

Zwischenzeitlich war in Hof ein Kriminalkommissar Diederichs aus Lüneburg eingetroffen. Er besaß Sondervollmachten des Herzogs von Braunschweig-Lüneburg zur Dingfestmachung der Diebe der Goldenen Tafel. Nachdem durch Zeugenaussagen die letzten Zweifel ausgeräumt waren, dass der Inquisit Nicol List in Hof und der gesuchte „Freiherr von der Mosel“ identisch sind, verständigten sich die Landesherren in Bayreuth/Hof, Lüneburg und Hartenstein, List gegen Kostenerstattung nach Celle auszuliefern.
List wurde unter dem Schutz eines 18-köpfigen militärischen Begleitkommandos zwischen Weihnachten und Neujahr 1698 nach Celle überführt. Unterwegs erfolgte die Zuladung von Lists Komplizen aus Weimar, Leipzig und Halle. Von Januar bis Mai 1699 wurden nochmals zahlreiche Verhöre durch die Inquisition durchgeführt. Da sich List keine Überlebenschance mehr ausrechnete, versuchte er eine Strafmilderung durch Aussagen als „Kronzeuge“ zu erreichen, wobei er nunmehr rückhaltslos seine Komplizen belastete.

Gegen ihn wurde ein neues Todesurteil gesprochen, in welchem ausdrücklich auf eine Strafmilderung aufgrund seiner Aussagebereitschaft verwiesen wurde: Anstelle der grausamsten Hinrichtungsart, des Räderns, Zerstoßen der Gliedmaßen mit eisernen Keulen von unten, Enthauptung mit einem Beil und Aufspießen des Kopfes auf einen Pfahl sowie Verbrennen des Körpers.

Nachdem ein letzter Fluchtversuch Lists, in der Nacht vor der Hinrichtung durch einen Fluchttunnel aus dem Gefängnis in Celle zu entkommen, durch Verrat eines Kumpanen gescheitert war, wurde List am 23. Mai 1699 mit fünf seiner Komplizen hingerichtet. Bereits am 21. März waren sechs Komplizen in Celle exekutiert worden. Weitere Hinrichtungen erfolgten bis 1705. Ein Teil der List'schen Komplizen konnte sich der Strafverfolgung dauerhaft entziehen, darunter der vermutliche Denunziant Lorenz Schöne und Lists Geliebte Anna von Sien.
Hinrichtung Lists und seiner Komplizen 1699 in Celle Stich, 1700

\section{Autor \\ Gerd Freitag \\ Chemnitz}

